\title{
REVIEW OF REHABILITATION OF ISCHAEMIC STROKE PATIENTS
}

\author{
MAHMOOD K ${ }^{1}$, HOSSAIN MS ${ }^{2}$, RAHMAN S
}

\begin{abstract}
:
Rehabilitation of stroke patient is a continuum, starting within days of onset and ending only when it no longer produces any positive effects. Although rehabilitation intervention is important during the acute phase of care, it is secondary to the activities involved in diagnosis and acute medical treatment. However, when a patient has a persisting major continuing impairment, such as hemiplegia with disabilities, the rehabilitation components of care quickly become the main focus of management. Stroke rehabilitation is always done by the patient, rather than to the patient. The major theme of rehabilitation is the enhancement of quality of life of stroke patient. The comprehensive rehabilitation management programme is characterized by a holistic approach and understanding the rehabilitation of the patients who sustain stroke. It is important because it involves virtually all elements of rehabilitation activity and all members of the rehabilitation team. In this article we aim to provide an overview of the area of rehabilitation in the management of ischemic stroke to improve the quality of service for the stroke patients.
\end{abstract}

Key words: Stroke, rehabilitation.

J Dhaka Med Coll. 2009; 18(2) : 169-173

\section{Introduction:}

Stroke is a clinical syndrome characterized by rapid onset of focal neurological deficit that lasts more than 24 hours or leads to death and has a presumed vascular cause. ${ }^{1}$ It is one of the major cause of death and disability. ${ }^{2}$ Approximately two thirds of stroke burden occurs in middle-aged subjects (45-69 years) and approximately two thirds occurs in developing region. ${ }^{3}$ In a study of Neuromedicine Department, Chittagong Medical College Hospital, majority of patients were from lower socio-economic class (46.2\% rural), lower level of education (47.2\% illiterate) and per capita income (Taka $1159+762 /$ month). ${ }^{4}$ After a stroke, $50 \%$ to $70 \%$ of patients regain functional independence. However, 15\% to 30\% of patients are permanently disabled and 20\% require institutional care at 3 months after onset. ${ }^{5}$ In a study of the UK Oxford shire Committee Project, the average risk of recurrence of stroke in 675 patients following their first stroke was $13 \%$ in the first year 15 times the risk in the general population and $4 \%$ in subsequent year. ${ }^{6}$ In patients who survived, the risk of recurrence was $30 \%$ by 5 years. ${ }^{6}$ This contributes the focuses on secondary prevention of stroke.

Rehabilitation is derived from the Latin word "Habile" means "to make able" and Latin prefix "Re" means "again". So rehabilitation means to make able again. A clear consensus exists that the purpose of rehabilitation is to limit the impact of stroke related brain damage on daily life by using a mixture of therapeutic and problem solving approaches. ${ }^{3,8,9}$ The high incidence and prevalence of stroke imply that stroke rehabilitation should be a major component of health service provision. A stroke is not simply a brain disease but affects the whole person and the family.

The key purposes of rehabilitation can be summarized as the "five Rs":

- Realization of potential: ensuring the duration of contact with therapy staff has been sufficiently long to observe a plateau phase in recovery.

- Re-enablement: focusing on promoting independence in daily living skills such as walking and dressing.

1. Assistant Professor, Department of Physical Medicine ,Dhaka Medical College, Dhaka.

2. Assistant Professor, Department of Physical Medicine, Dhaka Medical College, Dhaka.

3. Professor \& Head, Department of Physical Medicine ,Dhaka Medical College, Dhaka.

Correspondence: Dr. Khurshid Mahmood. 
- Resettlement: helping the person to leave hospital feeling safe, well supported, and confident.

- Role fulfillment: helping the person to reestablish their status and personal autonomy.

- Readjustment: helping the person to adapt to and accept a new lifestyle.

\section{Stroke recovery:}

Population based studies of stroke recovery have shown that the time taken to achieve best functional performance for mild, moderate, and severe strokes averages 8,13 , and 17 weeks respectively. ${ }^{10}$ The times vary considerably between individual patients, but these averages provide a useful guide for the duration of rehabilitation contact time.

An acute ischemic stroke lesion has a core of irrecoverable neurons surrounded by an ischemic penumbra of potentially viable neurons. Initial stroke recovery involves resolution of cerebral edema, ionic fluxes, and inflammatory processes followed by recruitment and reorganization of undamaged neural networks. Later recovery is adaptive to the new circumstances of residual impact of the stroke on daily life activities.

Rehabilitation is relevant to both phases. Early rehabilitation (first few months) uses techniques that seek to influence the potential for neuroplastic change, and later rehabilitation encourages adaptive responses and coping strategies based on educational and psychological theory.

Immediate rehabilitation in stroke unit

Stroke rehabilitation for most patients should start in hospital. The patients who are not admitted to hospital require rapid assessment by a specialist stroke rehabilitation team.

A Cochrane review provides conclusive evidence that patients who receive organized inpatient care (such as that provided by a multidisciplinary specialist team in a stroke unit) are more likely to be alive, independent, and living at home one year after stroke than patients who receive non-specialist care (such as that provided on medical wards). ${ }^{11}$ The benefits of organized stroke care were seen equally for older and young patients, male or female, and for all types of stroke. Stroke units, therefore, should not have restrictive admission criteria: the aim should be to treat every patient with a new stroke in a stroke unit.

The development of a rehabilitation stroke unit largely involves collecting together dispersed patients and staff into a single ward area. This is usually achievable at minimum additional cost, making the stroke rehabilitation unit a particularly attractive healthcare technology. Immediate admission to a stroke unit optimizes acute care such that complications arising after stroke-such as aspiration pneumonia or dehydration, which can contribute to additional brain damage-are minimized. This ensures a more favourable clinical context on which to start rehabilitation.

\section{Stroke unit:}

This unit involves coordinated care from a multidisciplinary team like neurologist, physiatrist with integration of nursing care; close involvement of carers in the rehabilitation process; technologist's expertise in stroke; and education about sign and symptoms of stroke complication along with clinical psychologist for depression, with training programmes for staff, patients, and carers. ${ }^{11,12}$

Key features of a stroke unit

- Staff with a specialist interest in stroke or rehabilitation.

- Routine involvement of carers in the rehabilitation process.

- Coordinated care from a multidisciplinary team, including meetings at least once each week.

- Information provided to patients and carers along with family member.

- Regular programmes of education and training.

The British Association of Stroke Physicians (www.basp.ac.uk) recommends that the 
minimum staffing levels on a stroke unit should be 1.0 consultant sessions per 10 beds; 8.0 trained or untrained nurses per 10 beds; 0.9 sessions of physiotherapy per bed; 0.7 sessions of occupational therapy per bed; 0.35 sessions of speech and language therapy per bed.

\section{What sort of physiotherapy?}

Physiotherapy after a stroke is valued highly by patients and two reviews provide strong evidence for its effectiveness. ${ }^{13,14}$ Which type of physiotherapy should be provided for which patient, however, remains uncertain. A review of 10 treatment intervention categories found strongest evidence for effectiveness for task orientated exercise training to restore balance and gait (for example, by practicing moving from sitting to standing). ${ }^{13}$

Upper limb impairment affects most patients at the time of the stroke, with persisting problems for between a half and three quarters of them. A review of trials of exercise therapy for upper limb impairment concluded that evidence was insufficient to inform clinical practice reliably. ${ }^{15}$ The review showed that more intensive exercise therapy is beneficial, but the researchers could not identify a subgroup of patients most likely to benefit. ${ }^{15}$

Language impairment will affect about a quarter of patients immediately after a stroke and will persist in about half of them. Usual practice is to offer speech and language therapy, but these treatments are poorly researched. 16

Cognitive impairment (including memory impairment), spatial neglect, and attention deficits are common, but the effectiveness of treatments is unclear because of insufficient research. Patients may not progress as well as first anticipated-the commonest reasons include cognitive impairment and mood disorders. These should, therefore, be routinely identified using standardized assessment instruments, such as the minimental state examination (cognitive impairment) and the hospital anxiety and depression scale (mood state).

Emerging approaches to stroke rehabilitation include motor imagery and robotics, and interest in progressive resistance strength training has re-emerged.

\section{How much physiotherapy?}

The average amount of one to one therapy provided to a patient is very small-about $6 \%$ of the working day. ${ }^{17} \mathrm{~A}$ review of inpatient and outpatient studies investigating intensity of therapy found that doubling the therapy significantly improved functional recovery but only a small amount: about one point 20 on the Barthel index scale. ${ }^{17}$ Although intensity of therapy is important, the organization and delivery of care may also be important. In a trial comparing coordinated, specialist, multidisciplinary care (nurses and therapists) with care given on a general ward-with the amount of care in both cases being similarthe coordinated care was associated with better outcomes. ${ }^{18}$

\section{Training of the care givers:}

A stroke causes a considerable burden of care, with up to three quarters of patients requiring help with daily living activities. ${ }^{19}$ Carer support is a key distinctive feature of organized, inpatient stroke services. ${ }^{11}$ A practical training programme for carers has been shown to be effective in decreasing burden and anxiety and depression among carers, and in improving psychological outcomes for patients ${ }^{20}$ and reducing costs. ${ }^{21}$

Training programme includes the following:

- Medication teaching

- Sign \& symptoms of common medical complication

- Instruction care of bladder catheter, respiratory equipment, and or feeding tubes

- Safety training

- Swallowing training speech therapy.

- Maintenance of nutrition and hydration

- Training in specific skills for which the patient needs assistance, and in methods of performing those skills (e.g., bed mobility, transfers, hygiene, dressing, others)

- Home exercise programme

- Others 


\section{Resettlement at home}

The timing of discharge from hospital is primarily determined by the level of support available at the patient's home for any functional disabilities. "Discharge" does not necessarily signify that maximum recovery from the stroke has occurred. The term "transfer of care" is, therefore, preferred as a more apt description, emphasizing the requirement to organize continuing contact with rehabilitation services.

A Cochrane review shows that for selected, moderately disabled stroke patients, early supported transfer of care using specialist stroke teams can reduce the length of hospital stay (on average by eight days), improve outcomes (reduction in risk of death or dependency by six patients per 100 patients treated), and improve patient satisfaction. ${ }^{22} \mathrm{~A}$ further Cochrane review has shown that stroke patients newly transferred home benefit from continuing contact with specialist therapy services, mainly in terms of less deterioration (seven patients do not deteriorate per 100 patients treated). ${ }^{23}$

A review of studies of community occupational therapy as a single discipline showed small additional benefits in functional independence and leisure activities. ${ }^{24}$ Single centre trials have reported a sustained improvement in outdoor mobility associated with community occupational therapy but not with community physiotherapy. The timely provision of aids, equipment, and environmental adaptations is regarded as an essential part of routine care but has not been well researched.

\section{Rehabilitation when the patient gets home}

Systematic reviews of qualitative ${ }^{25}$ and quantitative $^{26}$ studies of the experience of stroke recovery have described the diversity, complexity, and frequency of problems faced by patients and carers in the long term. Common problems include social isolation; restricted participation in leisure activities; delayed return to work; anxiety; depression; and distress.

Many studies have highlighted the importance of providing information during stroke recovery, but research suggests that the understanding of stroke and its consequences and the support available remain poor. A Cochrane review concluded that passive provision of information (for example, in the form of leaflets) is not associated with improved outcomes, whereas an educational approach (for example, some form of tutoring) might be effective. ${ }^{27}$ One of the difficulties is the complexity of the information needs and the paucity of effective management for some of the common conditions affecting stroke patients in the long term.

Despite the high prevalence of mood disorders, including depression, concern exists that recognition, assessment, $\&$ diagnosis of these conditions are poor. ${ }^{28}$ However, what should comprise routine care in this area is unclear. Psychotherapy has only a small treatment effect, and the effects of pharmacotherapy are uncertain except in the case of emotionalism, for which evidence exists that antidepressants can help.

Several service strategies to improve the long term outcomes have been investigated, including follow-up by specialist nurses, counselors, and family support workers. Provisional results from a systematic review of these studies indicate no associated improvement in health status or independence. 29

\section{Conclusion:}

The strongest evidence for effective stroke rehabilitation relates to better outcomes associated with specialist, coordinated, multidisciplinary teams, both during early inpatient recovery and for resettlement at home. At the present time we have a duty to provide our patients with the best possible stroke rehabilitation services and interventions, but we must never forget that we also owe our patients of the future and the opportunity to benefit from our continuing research enquiries. Considering all the multidimensional need of stroke patients, treatment facilities should be provided at 'stroke rehabilitation unit'. In Bangladesh, for comprehensive management of stroke patient, every medical college and medical university should have 'stroke rehabilitation unit' where the patient may get the necessary treatment. 


\section{References:}

1. Markus HS. Stroke: Cause and clinical feature. Medicine Intern 2005:32(10):36-40

2. Bonita R, Stewart AW, Beaglehole R. International trends in stroke mortality: 1970-1985.stroke 1990; 32:989-92

3. World Health Organization. The World Health Report 2002: Reducing Risks, Promoting Healthy life. Geneva, Switzerland: World Health Organization 2002.

4. Mollah AS, Rahman SW, Das KK, Hassanuzzaman M. Characteristics of patients admitted with stroke. Mymensingh Med J 2007 Jan; 16(1):2024

5. American Heart Association. Heart Disease and Stroke Statistics 2004 Update Dallas. Tex: American Heart Association; 2003.

6. Brown MM. Stroke: Prevention. Medicine Intern 2000; 4:57-58

7. Scottish Intercollegiate Guidelines Network (SIGN). Management of patients with stroke: rehabilitation, prevention and management of complications, and discharge planning. Edinburgh: SIGN, 2002. (SIGN publication No 64.)

8. Intercollegiate Working Party for Stroke. National clinical guidelines for stroke. London: Royal College of Physicians, 2004 (www.rcplondon.ac.uk/pubs/books/stroke/ index.htm).

9. Duncan PW, Zorowitz R, Bates B, Choi JY, Glasberg JJ, Graham GD, et al. Management of adult stroke rehabilitation care: a clinical practice guideline. Stroke 2005; 36:100-43.

10. Jorgensen H, Nakayama H, Raaschou H, ViveLarsen J, Stoier M, Olsen TS. Outcome and time course of recovery in stroke. Arch Phys Med Rehabil 1995; 76:406-12.

11. Stroke Unit Trialists' Collaboration. Organized inpatient (stroke unit) care for stroke. Cochrane Database Syst Rev 2001 ; (3):CD000197.

12. Langhorne P, Pollock A, for the Stroke Unit Trialists' Collaboration. What are the components of effective stroke unit care? Age Ageing 2002; 31:365-71.

13. Van Peppen RPS, Kwakkel G, Wood-Dauphinee S, Hendriks HJM, Van der Wees PJH, Dekker J. The impact of physical therapy on functional outcomes after stroke: what's the evidence? Clinical Rehabil 2004; 18:833-62.

14. Pollock A, Baer G, Pomeroy V, Langhorne P. Physiotherapy treatment approaches for the recovery of postural control and lower limb function following stroke. Cochrane Database Syst Rev 2007 (update in press).

15. Van der Lee JH, Smels IAK, Beckerman H, Lankhorst GJ, Wagenaar RC, Bouter LM. Exercise therapy for arm function stroke patient: a systematic review of randomised controlled trials. Clinical Rehabil 2001; 15:20-31.

16. Greener J, Enderby P, Whurr R. Speech and language therapy for aphasia following stroke. Cochrane Database Syst Rev 1999 ;(4):CD000425.

17. Kwakkel G, van Peppen R, Wagenaar RC, Dauphinee SW, Richards C, Ashburn A, et al. Effects of augmented exercise therapy time after stroke: a meta-analysis. Stroke 2004; 35:2529-36.

18. Kalra L, Dale P. Crome P. Improving stroke rehabilitation: a controlled study. Stroke 1993; 24:1462-7.

19. Han B, Haley W. Family care giving for patients with stroke. Stroke 1999; 30:1478-5.

20. Kalra L, Evans A, Perez I, Melbourn A, Patel A, Knapp M, et al. Training carers of stroke patients: randomized controlled trial. BMJ 2004; 328:1099104.

21. Patel A, Knapp M, Evans A, Perez I, Kalra L. Training care givers of stroke patients: economic evaluation. BMJ 2004; 328:1102-4.

22. Early Supported Discharge Trialists. Services for reducing duration of hospital care for acute stroke patients. Cochrane Database Syst Rev 2005 ;) 2):CD000443.

23. Outpatient Service Trialists. Therapy-based rehabilitation services for stroke patients at home. Cochrane Database Syst Rev 2003 ;( 1):CD002925.

24. Walker MF, Leonardi-Bee J, Bath P, Langhorne $\mathrm{P}$, Dewey $\mathrm{M}$, Corr $\mathrm{S}$, et al. Individual patient data meta-analysis of randomized controlled trails of community occupational therapy for stroke patients. Stroke 2004; 35:2226-32.

25. Mckevitt C, Redfern J, Mold F, Wolfe C. Qualitative studies of stroke: a systematic review. Stroke 2004; 35:1499-505.

26. Young J, Murray J, Forster A. Review of longerterm problems after disabling stroke. Rev Clin Gerontol 2003; 13:55-65.

27. Forster A, Smith J, Young J, Knapp P, House A, Wright J. Information provision for stroke patients and their caregivers. Cochrane Database Syst Rev $2001 ;$ (3):CD001919.

28. Hackett ML, Yapa C, Parag V, Anderson CS. Frequency of depression after stroke: a systematic review of observational studies. Stroke 2005; $36: 1330-40$.

29. Ellis G, on behalf of the Stroke Liaison Workers Collaboration. Meta-analysis of stroke liaison workers for patients and carers: results by intervention characteristic. Cerebrovasc Dis 2006; 21 (suppl 4): 120 . 